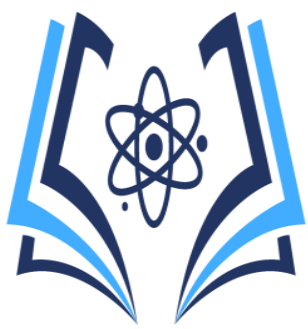

\title{
Rol de la enfermería en pacientes con complicaciones postoperatorias cardiovasculares
}

\author{
Nursing role in patients with postoperative cardiovascular \\ complications
}

\section{Rol de la enfermería en pacientes con complicaciones}

\author{
Mercedes del Rosario Acuña Acebo, Mg.Eii ${ }^{1}$ \\ Mercedes María Lucas Choez Mg. Sc ${ }^{2}$ \\ ${ }^{1}$ Universidad Estatal del Sur de Manabí, mercedes.acuna@unesum.edu.ec, https://orcid.org/0000-0001- \\ 8752-1930 \\ ${ }^{2}$ Universidad Estatal del Sur de Manabí, charya@hotmail.es, https://orcid.org/0000-0003-2001-7611
}

Contacto: mercedes.acuna@unesum.edu.ec

\begin{abstract}
Resumen
La enfermería es una de las disciplinas que más interviene en el proceso postoperatorio forma parte de una herramienta que permite al usuario participar de su propia atención, en donde la enfermería tiene como preocupación especial la necesidad del individuo, su provisión y ejecución de los roles de actuación de modo continuo para sostener la vida y la salud, recuperarse de la enfermedad o daño y adaptarse a sus efectos. El objetivo de esta investigación fue identificar el rol de la enfermera en los pacientes con complicaciones postoperatorias cardiovasculares. Se realizo una revisión bibliográfica esta búsqueda exhaustiva se realizó en las bases de datos como Scielo, Chocrane y Pubmed. La población estuvo conformada por 46 artículos de los cuales fueron seleccionados 9 artículos. En los resultados se evidencio que el rol asistencial es el que desempeña la enfermera/o ante las complicaciones postquirúrgicas cardiovasculares y que de estas complicaciones la que predomina son arritmias supraventriculares y crisis hipertensiva. Conclusión: el rol fundamental de la enfermera dependerá de la etapa en la cual se encuentre durante el periodo posoperatorio y de su respuesta ante el acto quirúrgico. Mediante la valoración del paciente, el profesional de enfermería determina

la estabilidad o la insatisfacción de las necesidades básicas planteadas a través de sus diferentes esferas de actuación, busca armonizar el cuidado favoreciendo la recuperación y la mejora en la calidad de vida aplicando sobre todo
\end{abstract}

el rol asistencial con el uso del pensamiento crítico y reflexivo.

Palabras claves: Actuación de enfermería, complicaciones posoperatorias, valoración en la etapa posoperatoria.

Abstract

Nursing is one of the disciplines that most intervenes in the postoperative process, it is part of a tool that allows the user to participate in their own care, where nursing has as a special concern the need of the individual, their provision and execution of the roles of acting continuously to sustain life and health, recover from illness or injury, and adapt to its effects. The objective of this research was to identify the role of the nurse in patients with postoperative cardiovascular complications. A bibliographic review was carried out. This exhaustive search was carried out in databases such as Scielo, Chocrane and Pubmed. The population consisted of 46 articles from which 9 articles were selected. The results show that the care role is that played by the nurse in the face of post-surgical cardiovascular complications and that of these complications the predominant one are supraventricular arrhythmias and hypertensive crisis. Conclusion: the fundamental role of the nurse will depend on the stage in which she is during the postoperative period and her response to the surgical act. By assessing the patient, the nursing professional determines the stability or dissatisfaction of the basic needs raised through its different spheres of action, seeks to harmonize care favoring recovery and improvement in quality of life, 
HIGÍA DE LA SALUD

applying above all the care role with the use of critical and reflective thinking.

Key words: Nursing performance, postoperative complications, assessment in the postoperative stage.

\section{Introducción}

En el marco de los esfuerzos desplegados por la Organización Mundial de la Salud (OMS) por reducir el número de defunciones de origen quirúrgico en todo el planeta, el departamento de Seguridad del Paciente de la OMS creó el programa La Cirugía Segura Salva Vidas. El programa pretende aprovechar el compromiso político y la voluntad clínica para abordar importantes cuestiones de seguridad, como las inadecuadas prácticas de seguridad anestésicas, las infecciones quirúrgicas evitables y la escasa comunicación entre los miembros del equipo quirúrgico. Se ha comprobado que estos son problemas habituales, que comportan riesgo mortal y podrían evitarse en todos los países y entornos. (1)

Las complicaciones postoperatorias son patologías que pueden presentarse ya sea en el postoperatorio inmediato o mediato. La índole es diversa, así como el grado en el que se compromete las funciones vitales del organismo. Ningún procedimiento quirúrgico, ni siquiera el más simple, está exento de riesgo, estas complicaciones se asocian con una mayor mortalidad hospitalaria. (2)

La enfermera cumple un rol vital y trascendental en el quehacer sanitario actuando de manera importante en lo que hoy se conoce como la medicina del futuro, es decir los cuidados de la vida y la salud en términos de prevención y promoción. Sin embargo, si analizamos el accionar de la enfermera veremos que la enfermera por ser inherente a su formación y por estar más cerca de la población y de los pacientes, tiene una vasta experiencia de vida personal y profesional relacionado a la promoción de la salud y prevención de enfermedades.

La intervención de la enfermera en la educación sanitaria del paciente es fundamental, tanto en la prevención como en la rehabilitación, para favorecer el control de los factores de riesgo, promover hábitos de vida saludables, disminuir la morbimortalidad y mejorar su calidad de vida. Los roles que la enfermera debe desempeñar deben ser completos, estas son formas de atención eficaz y primordial que garantizaran el correcto cuidado de los pacientes. (3)

Este proyecto se incorpora con las siguientes materias: Enfermería médico quirúrgico en la respectiva asistencia de enfermería a los pacientes con complicaciones cardiovasculares que son intervenidos quirúrgicamente $\mathrm{y}$ desarrollar actividades para la recuperación del usuario, con Psicología se relaciona ya que se
Periodo. Enero - Junio 2020

Vol. 2, Nro. 1, Publicado 2020-06-30 pueden determinar los problemas mentales y comportamientos que pueden llegar a desencadenar estas patologías, con Ética y Bioética para cumplir con los roles que se desempeñan, de manera eficaz siempre en el mando de lo correcto, con Psiquiatría relaciona la prevención de las posibles enfermedades mentales que se puedan suscitar, con Nutrición en la alimentación adecuada del paciente para la prevención o cuidado de su salud y las actividades físicas que tiene que realizar y en Investigación en enfermería en adquirir nuevos conocimientos que nos ayuden a desarrollar nuestra practica directa o indirecta de manera eficaz, además de la metodología de revisión bibliográfica en la que se basa este proyecto.

\section{Materiales y métodos}

Se plantea esta revisión bibliográfica en el cual se realizó una investigación documental, es decir, se hizo una recopilación de información ya existente sobre "El rol de la enfermera en las complicaciones postoperatorias cardiovasculares". Realizando una búsqueda exhaustiva mediante las bases de datos como Scielo, Chocrane y Pubmed.

Los cuales contienen muchos artículos que se relacionan con la investigación que realizamos, pero de todos ellos se escogieron los más relevantes para poder obtener los resultados esperados.

Estrategias de búsqueda

Las búsquedas realizadas en Scielo, Chocrane y Pubmed fueron envase a las "Complicaciones postoperatorias cardiovasculares". En las cuales las bases de datos utilizadas se encontraron 46 artículos relacionados, los mismos que se desglosaron de la siguiente manera:

- Scielo (15 artículos relacionados y 4 artículos que coincidieron),

- Chocrane (19 artículos relacionados y 2 artículos que coincidieron) y

- Pubmed (12 artículos relacionados y 3 artículos que coincidieron)

Dando un total de 46 artículos relacionados a las "complicaciones postoperatorias cardiovasculares" y 9 artículos que coincidieron con el tema mencionado.

Las palabras claves utilizadas fueron:

- En Scielo: "Complicaciones cardiovasculares (insuficiencia cardiaca aguda intervención quirúrgica)", "Cirugía cardiaca", "Complicaciones", "Complicaciones cardiovasculares", "infarto al miocardio", "Insuficiencia cardíaca aguda (ICA)".

- En Chocrane: "Complicaciones, accidentes cerebrovasculares", "Crisis hipertensivas".

- En Pubmed: "Postoperative cardiovascular complications", 
HIGIA DE LA SALUD

"Postoperative complications in cardiovascular surgery".

Filtros aplicados: Bibliografía, Libros y Documentos, Conferencia Clínica, Estudio Clínico, Ensayo Clínico, Artículo de Revista, Conferencia, Estudio Observacional, Revisión, Revisión Sistemática, en los últimos 5 años, inglés, español, Revistas de Enfermería.
Periodo. Enero - Junio 2020

Vol. 2, Nro. 1, Publicado 2020-06-30

\section{Resultados}

Se presentan los resultados de la revisión bibliográfica concerniente al rol de la en enfermera en complicaciones postoperatorias cardiovasculares, para lo cual se tomaron diversos estudios publicados desde el 2015 hasta el 2020 en revistas científicas indexadas regionales e internacionales.

\begin{tabular}{|c|c|c|c|c|c|}
\hline $\begin{array}{l}\text { AUTORES Y } \\
\text { NOMBRE } \\
\text { DEL } \\
\text { ESTUDIO }\end{array}$ & $\begin{array}{l}\text { BASE } \\
\text { DE } \\
\text { DATO }\end{array}$ & AÑ̃ & $\begin{array}{l}\text { PALABRA } \\
\text { CLAVE }\end{array}$ & $\begin{array}{l}\text { COMPLICACIONE } \\
\mathrm{S}\end{array}$ & $\begin{array}{l}\text { ROL } \\
\text { ENFERMERÍA }\end{array}$ \\
\hline $\begin{array}{l}\text { G Orlandova, } \\
\text { L Bello; } \\
\text { D Anchundia } \\
\text { Cirugía } \\
\text { cardíaca, } \\
\text { complicacione } \\
\text { s inmediatas } \\
\text { post } \\
\text { operatorias }\end{array}$ & Scielo & 2020 & $\begin{array}{l}\text { Complicaciones } \\
\text { cardiovasculares } \\
\text { (insuficiencia } \\
\text { cardiaca aguda } \\
\text { intervención } \\
\text { quirúrgica) }\end{array}$ & $\begin{array}{l}\text {-Crisis hipertensiva } \\
\text { post operatoria } \\
\text {-Insuficiencia } \\
\text { cardiaca aguda } \\
\text {-Arritmia } \\
\text {-(constituyen la } \\
\text { complicación que con } \\
\text { mayor frecuencia se } \\
\text { presenta en el post } \\
\text { operatorio.) }\end{array}$ & $\begin{array}{l}\text { Se aplicó el rol de } \\
\text { enfermería } \\
\text { investigativo ya que } \\
\text { el estudio fue } \\
\text { creado por un grupo } \\
\text { de enfermeras. }\end{array}$ \\
\hline $\begin{array}{l}\text { D. Cáceres, K. } \\
\text { Meneses, A. } \\
\text { Ramírez- } \\
\text { Sarmientoa, C. } \\
\text { Domínguez, } \\
\text { A. Bermonb, } \\
\text { A. Figueredof, } \\
\text { R. Duartea y } \\
\text { M Orozco } \\
\text { Levi. } \\
\text { Disfunción } \\
\text { muscular } \\
\text { respiratoria en } \\
\text { pacientes } \\
\text { llevados a } \\
\text { cirugía } \\
\text { cardiovascular } \\
\text {. }\end{array}$ & Scielo & 2016 & $\begin{array}{l}\text { Cirugía cardiaca; } \\
\text { Complicaciones; }\end{array}$ & $\begin{array}{l}\text {-Las complicaciones } \\
\text { respiratorias fueron } \\
\text { definidas según el } \\
\text { protocolo de manejo } \\
\text { del paciente sometido } \\
\text { a cirugía } \\
\text { cardiovascular de la } \\
\text { Fundación } \\
\text { Cardiovascular de } \\
\text { Colombia el cual se } \\
\text { basa en las } \\
\text { definiciones de "The } \\
\text { Society of Thoracy } \\
\text { Surgeons" (STS), } \\
\text { dentro de las cuales se } \\
\text { incluyen: ventilación } \\
\text {-Lla vánica prolongada } \\
\text { mecániar } \\
\text { (> 24 horas), } \\
\text {-El tromboembolismo } \\
\text { pulmonar (TEP) } \\
\text {-El neumotórax } \\
\text {-El hemotórax } \\
\text {-La neumonía } \\
\text { El derrame pleural }\end{array}$ & $\begin{array}{l}\text { No se aplicó ningún } \\
\text { rol de enfermería. }\end{array}$ \\
\hline 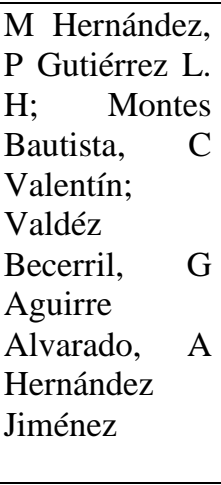 & Scielo & 2019 & $\begin{array}{l}\text { Complicaciones } \\
\text { cardiovasculares; } \\
\text { infarto al } \\
\text { miocardio }\end{array}$ & $\begin{array}{lr}\text { Para el } & \text { primer } \\
\text { estudio, se inyectaron } \\
\text { al paciente } 10 \mathrm{mCi} \text { de } \\
\text { Tc99m, y luego de } 45 \\
\text { minutos, re } \\
\text { adquirieron } \\
\text { imágenes } \\
\text { gamagráficas } \\
\text { SPECT CT gatillado, } \\
\text { las cuales fueron } \\
\text { interpretadas } \\
\text { analizadas por dos }\end{array}$ & $\begin{array}{l}\text { No se aplicó ningún } \\
\text { rol de enfermería. }\end{array}$ \\
\hline
\end{tabular}


ISSN 2773-7705

HIGÍA DE LA SALUD

Periodo. Enero - Junio 2020

\begin{tabular}{|c|c|c|c|c|c|}
\hline $\begin{array}{l}\text { Seguridad y } \\
\text { experiencia en } \\
\text { el uso del } \\
\text { oxígeno } \\
\text { hiperbárico en } \\
\text { el paciente con } \\
\text { infarto agudo } \\
\text { al miocardio } \\
\text { con elevación } \\
\text { del ST }\end{array}$ & & & & $\begin{array}{l}\text { expertos } \\
\text { Cardiología Nuclear y } \\
\text { Medicina Nuclear } \\
\text { cegados al tipo de } \\
\text { tratamiento al que fue } \\
\text { aleatorizado el } \\
\text { paciente. El segundo } \\
\text { estudio, a seis } \\
\text { semanas de distancia } \\
\text { del primero, se realizó } \\
\text { con protocolo de } \\
\text { estrés-reposo de un } \\
\text { solo día, para lo cual } \\
\text { se sometió a cada } \\
\text { paciente a estrés de } \\
\text { acuerdo con su clase } \\
\text { funcional y capacidad } \\
\text { física, ya fuera } \\
\text { farmacológico }\end{array}$ & \\
\hline $\begin{array}{l}\text { S Burgués } \\
\text { Insuficiencia } \\
\text { cardíaca aguda }\end{array}$ & Scielo & 2017 & $\begin{array}{l}\text { Insuficiencia } \\
\text { cardíaca aguda } \\
\text { (ICA) }\end{array}$ & $\begin{array}{l}\text {-Insuficiencia } \\
\text { cardiaca aguda (ICA) } \\
\text {-Síndromes } \\
\text { coronarios agudos. } \\
\text {-Hipertensión. } \\
\text {-Arritmias. } \\
\text {-Complicaciones } \\
\text { mecánicas agudas. } \\
\text {-Embolismo } \\
\text { pulmonar. }\end{array}$ & $\begin{array}{l}\text { Dentro de la } \\
\text { aplicación de los } \\
\text { roles de enfermería } \\
\text { encontramos en rol } \\
\text { asistencias debido a } \\
\text { que enfermería } \\
\text { asiste de manera } \\
\text { directa en el post } \\
\text { operatorio } \\
\text { pacientes a } \\
\text { insuficiencia con } \\
\text { cardiaca aguda, el } \\
\text { rol educativo ya que } \\
\text { enfermería educa a } \\
\text { los pacientes con } \\
\text { complicaciones } \\
\text { post quirúrgica } \\
\text { cardiovasculares } \\
\text { una vez que se } \\
\text { encuentran en su } \\
\text { etapa la } \\
\text { recuperación en el } \\
\text { potes operatorios, } \\
\text { para encaminar al } \\
\text { paciente a que su } \\
\text { salud se recupere y } \\
\text { por ende se dé su } \\
\text { alta, y el rol } \\
\text { investigativo } \\
\text { debido a que } \\
\text { enfermería } \\
\text { investiga el uso de } \\
\text { técnicas } \\
\text { farmacológicas( } \\
\text { como do } \\
\text { ultrafiltración) para } \\
\text { la disminución de } \\
\text { dicha afección en el } \\
\text { caso de que los } \\
\text { pacientes } \\
\text { requieran }\end{array}$ \\
\hline
\end{tabular}


ISSN 2773-7705

HIGÍA DE LA SALUD

Periodo. Enero - Junio 2020

\begin{tabular}{|c|c|c|c|c|c|}
\hline $\begin{array}{l}\text { Wai-Tat } \\
\text { Wong, } \\
\text { Veronica KW } \\
\text { Lai, Yee Eot } \\
\text { Chee,Anna } \\
\text { Lee } \\
\text { Cuidado } \\
\text { cardiaco } \\
\text { rápido para } \\
\text { pacientes } \\
\text { adultos } \\
\text { sometidos a } \\
\text { cirugía } \\
\text { cardíaca }\end{array}$ & Cochra & 2016 & $\begin{array}{l}\text { Complicaciones, } \\
\text { accidentes } \\
\text { cerebrovasculares }\end{array}$ & $\begin{array}{l}\text {-Infarto al miocardio } \\
\text {-Accidentes } \\
\text { cerebrovasculares }\end{array}$ & $\begin{array}{l}\text { No se aplicó ningún } \\
\text { rol de enfermería }\end{array}$ \\
\hline $\begin{array}{l}\text { AE. Delgado } \\
\text { Martína, J. } \\
\text { Sánchez } \\
\text { Lópeza, HJ. } \\
\text { Muñoz } \\
\text { Beltrána } \\
\\
\text { Rol de } \\
\text { enfermería en } \\
\text { el manejo de } \\
\text { las crisis } \\
\text { hipertensivas }\end{array}$ & $\begin{array}{l}\text { Cochra } \\
\text { ne }\end{array}$ & 2016 & $\begin{array}{l}\text { Crisis } \\
\text { hipertensivas. }\end{array}$ & $\begin{array}{l}\text {-Crisis hipertensivas } \\
\text {-Arritmias } \\
\text {-Taquicardia }\end{array}$ & 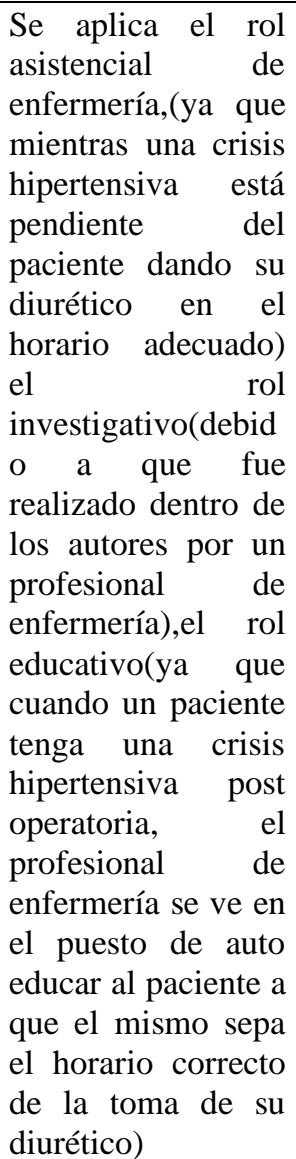 \\
\hline $\begin{array}{l}\text { Regi } \\
\text { Freeman, } \\
\text { Erika } \\
\text { Koerner, } \\
\text { Courtney } \\
\text { Clark, Kathy } \\
\text { Halabicky } \\
\text { Manejo } \\
\text { cuidado } \\
\text { posoperatorio } \\
\text { del trasplante } \\
\text { cardíaco }\end{array}$ & $\begin{array}{l}\text { PubMe } \\
\text { d }\end{array}$ & 2016 & $\begin{array}{l}\text { Postoperative } \\
\text { cardiovascular } \\
\text { complications }\end{array}$ & $\begin{array}{l}\text {-Taquicardias } \\
\text { Supraventriculares } \\
\text { (TSV) } \\
\text {-Crisis Hipertensiva } \\
\text { Postoperatoria. }\end{array}$ & $\begin{array}{l}\text { Los roles que se } \\
\text { mencionan son: El } \\
\text { asistencias } \\
\text { enfermeras deben } \\
\text { estar alerta ante } \\
\text { cualquier } \\
\text { complicación que } \\
\text { se puede llegar a } \\
\text { presentar) y El } \\
\text { administrativo (La } \\
\text { enfermera debe } \\
\text { estar alerta si se } \\
\text { requieren exámenes } \\
\text { complementarios o } \\
\text { debe estar presente } \\
\text { en alguno de ellos.) }\end{array}$ \\
\hline
\end{tabular}


ISSN 2773-7705

HIGÍA DE LA SALUD

Periodo. Enero - Junio 2020

\begin{tabular}{|c|c|c|c|c|c|}
\hline $\begin{array}{l}\text { Carol R } \\
\text { Wade 1, Krist } \\
\text { ine } \\
\text { Reith, JoAnn } \\
\text { Hoffman } \\
\text { Sikora, Sharo } \\
\text { n M Augustine } \\
\\
\text { Atención de } \\
\text { enfermería } \\
\text { postoperatoria } \\
\text { del receptor de } \\
\text { trasplante } \\
\text { cardíaco }\end{array}$ & $\begin{array}{l}\text { PubMe } \\
\text { d }\end{array}$ & 2016 & $\begin{array}{l}\text { Postoperative } \\
\text { cardiovascular } \\
\text { complications }\end{array}$ & $\begin{array}{l}\text {-Denervación } \\
\text { cardíaca } \\
\text {-Disfunción del } \\
\text { aloinjerto } \\
\text {-Manejo de arritmias } \\
\text {-Rechazo } \\
\text { Inmunosupresión } \\
\text {-Complicaciones } \\
\text { infecciosas }\end{array}$ & $\begin{array}{l}\text { El rol que se } \\
\text { menciona es el rol } \\
\text { asistencial ya que se } \\
\text { debe asistir a estos } \\
\text { pacientes en las } \\
\text { complicaciones } \\
\text { presentadas. }\end{array}$ \\
\hline $\begin{array}{l}\text { Samantha } \\
\text { Hoy 1, Jason } \\
\text { Frisbee } \\
\text { Complicacion } \\
\text { es frecuentes } \\
\text { del trasplante } \\
\text { cardíaco } \\
\text { posoperatorio }\end{array}$ & $\begin{array}{l}\text { PubMe } \\
\text { d }\end{array}$ & 2018 & $\begin{array}{l}\text { Postoperative } \\
\text { complications in } \\
\text { cardiovascular } \\
\text { surgery }\end{array}$ & -Arritmias & $\begin{array}{l}\text { No se aplicaron } \\
\text { Roles. }\end{array}$ \\
\hline
\end{tabular}

\section{Análisis de los resultados}

En la revisión bibliográfica realizada encontramos 49 artículos relacionados con nuestro tema de investigación de los cuales solo 9 de ellos tuvieron relevancia con nuestro tema "Complicaciones postoperatorias cardiovasculares" de nuestro Proyecto Integrador de Saberes (PIS). Los roles de la enfermera en las complicaciones del paciente en el post operatorio es el tema que generalmente vamos a tratar en esta investigación, por el cual además de las complicaciones postoperatorias que se presentan también se hizo énfasis en los roles que desempeña la enfermera en esta etapa ya que ella es la encargada del cuidado y atención de estos. Por lo que la enfermera debe ejecutar sus cuatro roles, como lo son: el rol asistencial (Es el que mayormente se realiza ya que el personal de enfermería es el que va a estar en constante cuidado del paciente en la etapa postoperatoria al igual que en las otras, así atendiendo sus necesidades básicas y ayudándolo a mejorar su salud); el rol educativo (la mayoría de los pacientes no tienen conocimientos de los procedimientos que se les realizan, es por ello que este rol debe cumplirse, ya que al educar a los pacientes contribuye a que las estas personas pierdan en ciertos casos el temor y la ansiedad que estos generan en los usuarios, al igual que debe educar al cuidador del paciente y prepararlo para cuando el aquel reciba el alta médica); rol administrativo (La enfermera también se encargada de evaluar, organizar, promover y supervisar la calidad de los cuidados de enfermería, el personal que este bajo su supervisión debe actuar de manera que tanto los pacientes como los familiares se encuentren satisfechos) y por último el rol investigativo ( $\mathrm{Si}$ bien es cierto el agravamiento de salud puede ser un gran precursor de que los pacientes puedan presentar cuadros de trastornos psicológicos debido a los procedimientos realizados, por lo que la enfermera deberá investigar el comportamiento de estos para saber cómo actuar en estos casos y ayudar a restablecer su salud mental).

Por otra parte como se puede evidenciar en nuestra revisión bibliográfica realizada en las bases de datos ya antes mencionadas (Scielo, Chocrane y Pubmed) las complicaciones post operatorias cardiovasculares que mayormente se presentan con frecuencia son las arritmias supraventriculares, además de la hipertensión, luego de haber digitado en las bases de datos las siguientes palabras claves: "Complicaciones cardiovasculares (insuficiencia cardiaca aguda intervención quirúrgica)", "Cirugía cardiaca", "Complicaciones", "Complicaciones cardiovasculares", "infarto al miocardio", "Insuficiencia cardíaca aguda (ICA)", "Complicaciones, accidentes cerebrovasculares", "Crisis hipertensivas", "Postoperative cardiovascular complications", 
HIGIA DE LA SALUD

"Postoperative complications in cardiovascular surgery".

\section{Conclusiones}

- De acuerdo a la revisión sistemática realizada se encontró que las principales complicaciones cardiovasculares en la etapa postoperatoria fueron: Taquicardias supraventriculares, paro cardiorrespiratorio y crisis hipertensiva postoperatoria, estas presentes en
Periodo. Enero - Junio 2020 Vol. 2, Nro. 1, Publicado 2020-06-30 pacientes con falla cardiaca que han sido intervenidos de angioplastia.

- El rol de la enfermera que más desempeña es asistencial porque desarrolla actividades que preservan la dignidad e integridad del paciente con el fin de mejorar la salud de este, realizando intervenciones de enfermería y respondiendo a sus necesidades.

\section{Colaboradores en la Investigación:}

Estudiantes de la Carrera de Enfermería de la Universidad Estatal del Sur de Manabí.

Cedeño Piloso Gema Yulexy, Cevallos Choez Sabina Catherine, Chaguay Holguín José Gabriel, Jara Moran Lady Yessenia, Marcillo Baque Evelyn Dayana, Mero Mero María Elena, Ortega Barrezueta Marina Isabel, Ospina Ramírez Nila Alejandra, Parrales Gómez Hugo Ernesto, Quimís Quijije Johana Elizabeth.

\section{BIBLIOGRAFÍA}

OMS. MEDICALCENTER. [Online].; 2018 [cited 2020 AGOSTO 11. Available from: https://apps.who.int/iris/bitstream/handle/10665/44233/9789243598598_spa.pdf;jsessionid=FFD664900 49ED776E643E8D572F74489?sequence=1.

Martinez J. es.slideshare.net. [Online].; 2017 [cited 2020 Agosto 18. Available from: https://es.slideshare.net/Juliiom13/complicaciones-postoperatorias-47204958.

Carlos S. www.fbbva.es. [Online].; 2018 [cited $2020 \quad 07 \quad 18 . \quad$ Available from: https://www.fbbva.es/microsites/salud_cardio/mult/fbbva_libroCorazon_cap69.pdf.

Jorge Olmos Salamanca PVMM. www.sciencedirect.com. [Online].; 2016 [cited 202007 18. Available from: https://www.sciencedirect.com/science/article/pii/S012173721600011X.

Torres M. files.sld.cu/enfermeria-pediatria. [Online].; 2017 [cited 202007 18. Available from: https://files.sld.cu/enfermeria-pediatria/files/2011/03/cuidados-de-enf-inscardiaca.pdf.

Fabricio S. www.revista-portalesmedicos.com. [Online].; 2017 [cited 202007 18. Available from: https://www.revista-portalesmedicos.com/revista-medica/cuidados-de-enfermeria-insuficiencia-

cardiaca/.

codem. www.codem.es. [Online].; 2018 [cited 2020 Agosto 11. Available from: http://www.codem.es/Adjuntos/CODEM/Documentos/Informaciones/Publico/9e8140e2-cec7-4df7-8af98843320f05ea/8c06b7e5-ca29-40c6-ab63-f84959a87362/c618e862-974d-4faf-8093-

66eae984e3da/TRABAJO_CONGRESO_GRAFICA_AJUSTADA.pdf.

fundacionindex. www.fundacionindex.com. [Online].; 2019 [cited 2020 Agosto 11. Available from: http://www.fundacionindex.com/gomeres/?p=626.

imss.mx. www.imss.gob.mx. [Online].; 2017 [cited 2020 Agosto 18. Available from: http://www.imss.gob.mx/sites/all/statics/guiasclinicas/591GRR.pdf.

medwave. www.medwave.cl. [Online].; 2018 [cited 2020 AGOSTO 11. Available from: https://www.medwave.cl//ink.cgi/Medwave/Reuniones/Anestesiologia/Octubre/2438.

revcardiologia. www.www.revcardiologia.sld.cu.sld.cu. [Online].; 2019 [cited 2020 Agosto 11. Available from: http://www.revcardiologia.sld.cu/index.php/revcardiologia/article/view/633/html_36.

medlineplus. medlineplus.gov. [Online].; 2019 [cited 2020 Agosto 11. Available from: https://medlineplus.gov/spanish/ency/article/007639.htm.

Sweis RN. www.msdmanuals.com. [Online].; 2018 [cited 2020 Agosto 18. Available from: https://www.msdmanuals.com/es/professional/trastornos-cardiovasculares/enfermedadcoronaria/generalidades-sobre-los-s\%C3\%ADndromes-coronarios-agudos-sca\#v935311_es.

my-ekg. www.my-ekg.com. [Online].; 2018 [cited 2020 Agosto 11. Available from: https://www.myekg.com/arritmias-cardiacas/taquicardias-ventriculares/taquicardias-

ventriculares.html\#: :text=Taquicardia\%20Ventricular\&text=Las\%20taquicardias\%20ventriculares\%20 (TV)\%20son,con\%20una\%20frecuencia\%20cardiaca\%20elevada.

Mayoclinic. www.mayoclinic.org. [Online].; 2018 [cited 2020 Agosto 18. Available from: https://www.mayoclinic.org/es-es/diseases-conditions/ventricular-tachycardia/symptoms-causes/syc-

20355138. 
my-ekg. www.my-ekg.com. [Online].; 2019 [cited 2020 Agosto 11. Available from: https://www.myekg.com/arritmias-cardiacas/taquicardias-supraventriculares.html.

Mayoclinic. www.mayoclinic.org. [Online].; 2019 [cited 2020 Agosto 18. Available from: https://www.mayoclinic.org/es-es/diseases-conditions/supraventricular-tachycardia/symptomscauses/syc-20355243.

saludsavia. www.saludsavia.com. [Online].; 2019 [cited 2020 Agosto 11. Available from: https://www.saludsavia.com/contenidos-salud/enfermedades/paro-cardiorrespiratorio.

Mayoclinic. www.mayoclinic.org. [Online].; 2017 [cited 2020 Agosto 18. Available from: https://www.mayoclinic.org/es-es/diseases-conditions/sudden-cardiac-arrest/symptoms-causes/syc20350634.

fundaciondelcorazon. fundaciondelcorazon.com. [Online].; 2019 [cited 2020 Agosto 11. Available from: https://fundaciondelcorazon.com/informacion-para-pacientes/enfermedades-

cardiovasculares/tromboembolismo-pulmonar.html.

Cuidateplus. cuidateplus.marca.com. [Online]; 2017 [cited 2020 Agosto 18. Available from: https://cuidateplus.marca.com/enfermedades/respiratorias/embolia-pulmonar.html.

revespcardiol. www.revespcardiol.org. [Online]; 2018 [cited 2020 Agosto 11. Available from: https://www.revespcardiol.org/es-insuficiencia-cardiaca-aguda-epidemiologia-factores-articulo-

S0300893214006563\#: :text=La\%20insuficiencia\%20cardiaca\%20aguda\%20(ICA,hospitalizaci\%C3\% B3n\%20del\%20paciente1\%E2\%80\%933.

Empendium. empendium.com. [Online].; 2018 [cited 2020 Agosto 18. Available from: https://empendium.com/manualmibe/chapter/B34.II.2.19.2.

elsevier. www.elsevier.es. [Online].; 2018 [cited 2020 Agosto 11. Available from: https://www.elsevier.es/es-revista-medicina-integral-63-articulo-manejo-crisis-hipertensivas-

13045397\#: :text=Crisis\%20hipertensiva.,Emergencia\%20hipertensiva.

Geosalud. www.geosalud.com. [Online].; 2018 [cited 2020 Agosto 18. Available from: https://www.geosalud.com/hipertension/crisishipertensiva.htm\#: :text=La\%20droga\%20de\%20elecci\% C3\%B3n\%20para,e\%20impredecible\%20est\%C3\%A1n\%20totalmente\%20contraindicados\%20.

G Orlandova LB. Scielo. [Online].; 2020 [cited 202009 08. Available from: http://scielo.sld.cu/scielo.php?script=sci_arttext\&pid=S2218-

$36202020000200293 \& \operatorname{lng}=$ pt\&nrm=iso\&tlng=pthttp://scielo.sld.cu/scielo.php?script=sci_arttext\&pid=S 2218-

36202020000200293\&lng=pt\&nrm=iso\&tlng=pthttp://scielo.sld.cu/scielo.php?script=sci_arttext\&pi.

D. Cáceres KMARSCDABAFRDyMOL. Scielo. [Online].; 2016. Available from: http://www.scielo.org.co/scielo.php?script=sci_abstract\&pid=S0120-

$56332016000500420 \& \operatorname{lng}=$ en\&nrm=iso\&tlng=es.

M Hernández PGLH, Monte Bautista CV, Valdéz Becerril GAAAHJ. Scielo. [Online].; 2016. Available from: http://www.scielo.org.mx/scielo.php?script=sci_arttext\&pid=S0301-696X2018000300231.

$\begin{array}{llllll}\text { Burgués } & \text { S. } & \text { Scielo. } & \text { [Online].; } & 2017 . & \text { Available }\end{array}$ http://www.scielo.edu.uy/scielo.php?script=sci_arttext\&pid=S1688-04202017000300370.

Wai-Tat Wong VKLYECL. Cochranelibrary. [Online].; 2016 [cited 20200908 . Available from: https://www.cochranelibrary.com/cdsr/doi/10.1002/14651858.CD003587.pub3/full/es.

AE. Delgado Martína JSLHMB. Cochrane. [Online].; 2003 [cited 2020. Available from: https://www.elsevier.es/es-revista-medicina-integral-63-articulo-manejo-crisis-hipertensivas-13045397.

Regi Freeman EK,CC,KH. pubmed. [Online].; 2016 [cited 2020 Septiembre 08. Available from: https://pubmed.ncbi.nlm.nih.gov/27254638/.

Carol R Wade KKRJHSSMA. pubmed. [Online].; 2004 [cited 2020 Septiembre 08. Available from: https://pubmed.ncbi.nlm.nih.gov/14974522/.

Samantha Hoy JF. PubMed. [Online]; 2018 [cited 2020 Septiembre 08. Available from: https://pubmed.ncbi.nlm.nih.gov/30153181/. 